ARTICLE

\title{
Reconfigurable multi-component micromachines driven by optoelectronic tweezers
}

\author{
Shuailong Zhang (1) 1,2,3,8,9, Mohamed Elsayed (1) 1,3, Ran Peng4 ${ }^{4}$ Yujie Chen (1) 5, Yanfeng Zhang5 , Jiaxi Peng 1,2,3, \\ Weizhen Li ${ }^{6}$, M. Dean Chamberlain ${ }^{1,2,3}$, Adele Nikitina ${ }^{1}$, Siyuan $\mathrm{Yu}^{5,7}$, Xinyu Liu (i) ${ }^{4}$, Steven L. Neale (1) ${ }^{6} \&$ \\ Aaron R. Wheeler (1D) 1,2,3凶
}

There is great interest in the development of micromotors which can convert energy to motion in sub-millimeter dimensions. Micromachines take the micromotor concept a step further, comprising complex systems in which multiple components work in concert to effectively realize complex mechanical tasks. Here we introduce light-driven micromotors and micromachines that rely on optoelectronic tweezers (OET). Using a circular micro-gear as a unit component, we demonstrate a range of new functionalities, including a touchless microfeed-roller that allows the programming of precise three-dimensional particle trajectories, multi-component micro-gear trains that serve as torque- or velocity-amplifiers, and microrack-and-pinion systems that serve as microfluidic valves. These sophisticated systems suggest great potential for complex micromachines in the future, for application in microrobotics, micromanipulation, microfluidics, and beyond.

\footnotetext{
${ }^{1}$ Institute of Biomedical Engineering, University of Toronto, Toronto, ON, Canada. ${ }^{2}$ Department of Chemistry, University of Toronto, Toronto, ON, Canada. ${ }^{3}$ Donnelly Centre for Cellular and Biomolecular Research, University of Toronto, Toronto, ON, Canada. ${ }^{4}$ Department of Mechanical and Industrial Engineering, University of Toronto, Toronto, ON, Canada. ${ }^{5}$ State Key Laboratory of Optoelectronic Materials and Technologies, School of Electronics and Information Technology, Sun Yat-Sen University, Guangzhou, China. ${ }^{6}$ James Watt School of Engineering, University of Glasgow, Glasgow, UK. ${ }^{7}$ Photonics Group, Merchant Venturers School of Engineering, University of Bristol, Bristol, UK. ${ }^{8}$ Present address: Beijing Advanced Innovation Center for Intelligent Robots and Systems, Beijing Institute of Technology, Beijing, China. ${ }^{9}$ Present address: School of Mechatronical Engineering, Beijing Institute of Technology,

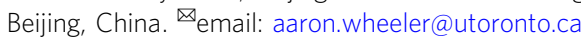


$\mathrm{M}$ icromotors are submillimeter particles or assemblies of particles that can convert energy into motion. There are many examples of micromotors in the literature, which can be classified on the basis of the type of energy that drives them, including magnetic ${ }^{1-5}$, acoustic $c^{6,7}$, electric ${ }^{8,9}$, and optical energies ${ }^{10-16}$. There is less consensus on the definition of the term "micromachine." A working definition used here is a submillimeter system employing multiple components whose motions are coupled together to drive useful mechanical work. Although the term is used frequently in the literature, there are very few reports that satisfy this definition.

Micromotors (and potentially, micromachines) that are driven by light are particularly appealing to consider, given the maturity and diversity of beam-modulation components that can be used $^{17-20}$, and light-driven micromotors can be subclassified into three categories based on their operating principles. The first category, opto-mechanical micromotors, relies on the interaction between light and photosensitive, mechanically responsive materials such as liquid-crystalline elastomers ${ }^{21-26}$. While this form of micromotor is an interesting and growing research topic, apart from demonstrations of simple functions, there are substantial material limitations that have precluded the use of these systems for many practical applications. The second category, opto-chemical micromotors, relies on photochemical reactions such as photochromic, photothermal, and photocatalytic effects to generate propulsion forces ${ }^{27-31}$. Opto-chemical micromotors have been demonstrated for interesting applications, but require highly specific operating conditions, including motors formed from photoactive materials and an environment that contains photochemically active reagents. The third category, optical micromotors, relies on direct manipulation of (otherwise unremarkable) microscale objects by illumination with light-the most prevalent example being optical tweezers (OTs) $)^{17-20,32-37}$. OT leverages the forces that arise from focusing light into steep intensity gradients, which permits fine and noninvasive control and actuation of microparticles suspended in air or liquid ${ }^{19,20}$. To date, there have been many successful demonstrations of OTactuated micromotors that can carry out complex mechanical operations, including microfluidic pumping ${ }^{38,39}$, directed tissue growth $^{10}$, and precise cell/particle translation ${ }^{12,40}$.

Although optical micromotors based on OT have been used for many impressive demonstrations and have many unique advantages (such as nanometer-level spatial precision ${ }^{12}$ ), OT is not a panacea for all applications. For example, OT generates forces on the order of picoNewtons $\left(10^{-12} \mathrm{~N}\right)$, which is useful for manipulating small particles (with sizes $<30 \mu \mathrm{m}$ ), but not large ones. This influences the design, structure, materials and tools used to form/ fabricate the micromotors, and sets certain limits on their applications, particularly for those involving larger objects. In addition, while OT can be used to manipulate multiple micromotors in parallel, this functionality requires specialized and expensive beammodulation hardware and control software ${ }^{12,19,20,41}$. This capacity was particularly important for the work described here, in which we sought to develop complex micromachines, which requires facile and robust methods for controlling many components in parallel, causing them to interact with each other.

Here, we introduce optical micromotors that are operated and controlled by optoelectronic tweezers $(\mathrm{OET})^{42-45}$. OET is an optical micromanipulation technology that relies on photoconductive substrates that are typically insulating, but become conductive upon illuminating with light. By projecting illuminated and dark regions onto the photoconductive substrate, lightactivated virtual electrodes can be formed in OET, which induce nonuniform electric fields producing DEP forces ${ }^{42-52}$. OET is capable of generation of forces on the order of nanoNetwons $\left(10^{-9} \mathrm{~N}\right)^{53}$, which permits the manipulation of objects with sizes
$>100 \mu \mathrm{m}^{54,55}$. In addition, it is particularly straightforward to use OET for parallel manipulation ${ }^{41-45}$, simply by projecting movies of moving shapes into a microscope. Recently, OET was used to control discrete microrobots capable of translating secondary payloads ${ }^{56}$. We are enthusiastic about this previous report, but note that each microrobot in the previous study achieved its functionality independently.

In this work, we sought to move beyond the single micromotor (or microrobot) to form complex, multicomponent, reconfigurable micromachines. Specifically, we describe a unit componenta light-driven micromotor that can be precisely rotated and translated-and characterize its behavior in diverse environments. We then describe how the motions of multiple micromotors can be coupled together, effectively forming light-driven, multicomponent micromachines, that can serve as $3 \mathrm{D}$ particle manipulators, torque and speed multipliers, and microfluidic valves. These examples are quite unique relative to what has been demonstrated previously, with potential utility for applications in hydrodynamics, microrobotics, particle/cell manipulation, and beyond.

\section{Results and discussion}

OET control of micromotors. The unit component of the lightdriven micromachines described here is a circular micromotor (or micro-gear) that features eight teeth arranged symmetrically around a disc with a hole in the center. Figure la is a representative scanning electron microscope (SEM) image of a microgear, which has a diameter of $200 \mu \mathrm{m}$ and a thickness of $60 \mu \mathrm{m}$ (Supplementary Note 1 and Supplementary Fig. 1). Figure 1b illustrates how micro-gears are formed by photolithography, followed by release from a sacrificial layer. As illustrated in Fig. 1c, after formation, micro-gears are transferred to a microscopy-based OET system in which light from a digital micromirror device (DMD) pattern illuminator is focused onto an OET device bearing a photoconductive layer [in this case, hydrogenated amorphous silicon (a-Si:H)] to exert the actuation force. Conditions were chosen such that DEP forces are negative, which serves to push the micro-gears into regions that are not illuminated. There are many examples in the OET literature ${ }^{43,49,50,57}$ that use simple hollow light patterns (e.g., a doughnut) to manipulate the positions of particles in this manner. Here, we adapted an approach described previously ${ }^{56}$ for the manipulation of microrobots, in which the light patterns are used as opto-mechanical components. For example, as shown in Fig. 1d, a standard set of components used here is an optical axle and an optical ring spanner with teeth designed to interface with those of the micro-gear. By rotating (Supplementary Movie 1) or translating (Supplementary Movie 2) the optical components, the micro-gears can be made to rotate or translate, as well (described in detail in Supplementary Note 2). Various combinations of rotation and translation enable manipulation of many micro-gears in parallel (Supplementary Movie 3 and Fig. 1e-h), a key property that is leveraged in the micromachines described below.

Prior to exploration of the capacity to use multiple micro-gears to form micromachines, we paused to evaluate the effect of ringspanner geometry on micro-gear behavior. Specifically, ring spanners with ring thicknesses of $0,10,30$, and $60 \mu \mathrm{m}$ (Fig. 2a-d) were applied to manipulate micro-gears-when manipulated at low velocities, the micro-gears behaved as programmed. At high velocities, however, the micro-gears can no longer follow the light pattern and the operations failed. We call the condition that divides these phenomena the maximum velocity, which is plotted for rotation and translation in Fig. 2e, f. As shown, thick rings allow for much higher velocities, while micro-gears driven by thin rings fail more readily at low velocities. This result is 

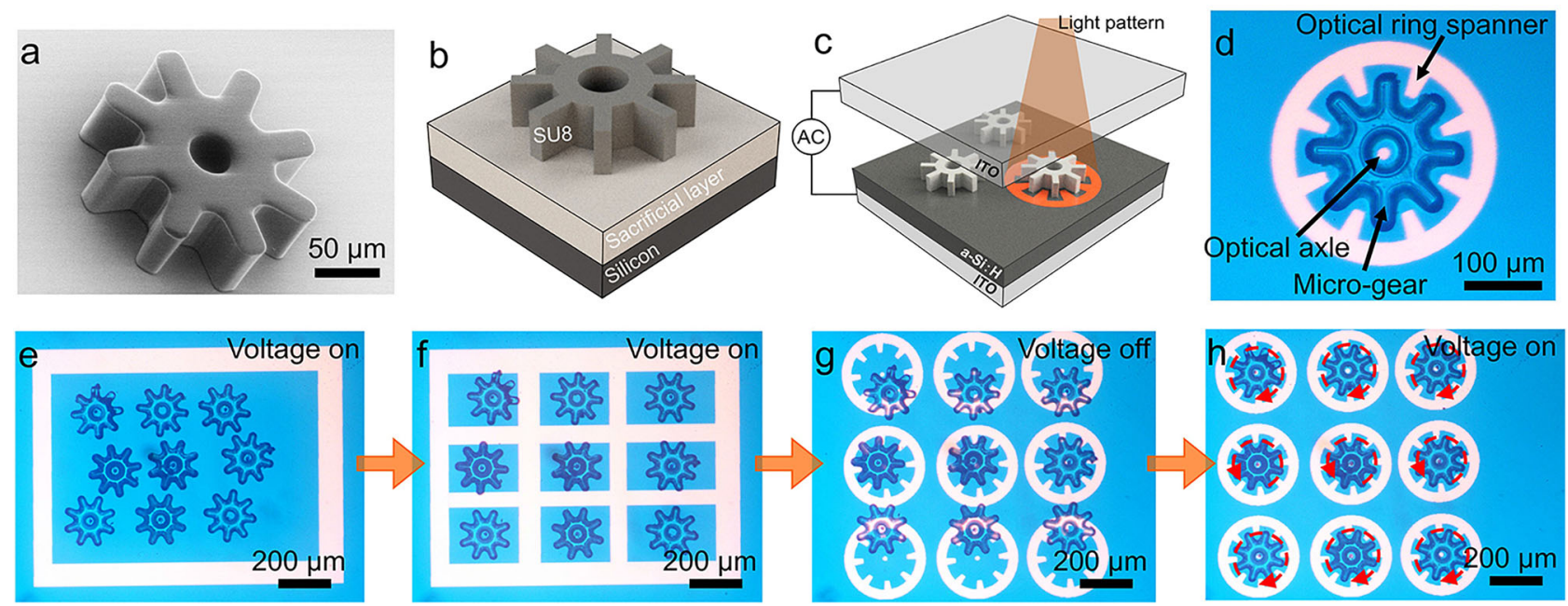

Fig. 1 OET control of micromotors. a SEM image of a 200- $\mu$ m-diameter micro-gear. b 3D schematic structure of a micro-gear prior to release. c Schematic depicting the principle of manipulating a micro-gear in an OET device. $\mathbf{d}$ Microscope image of a micro-gear being manipulated in an OET device. e-h Frames from Supplementary Movie 3 illustrating the step-by-step process (represented by orange arrows) of manipulating nine micro-gears such that they can be simultaneously rotated. The red-dashed arrows in (h) indicate that the micro-gears are rotating clockwise at $60 \%$ s in the upper row, counterclockwise at $60 \% \mathrm{~s}$ in the mid row, and clockwise at $120 \% \mathrm{~s}$ in the bottom row. This parallel manipulation process requires $\sim 30 \mathrm{~s}$.
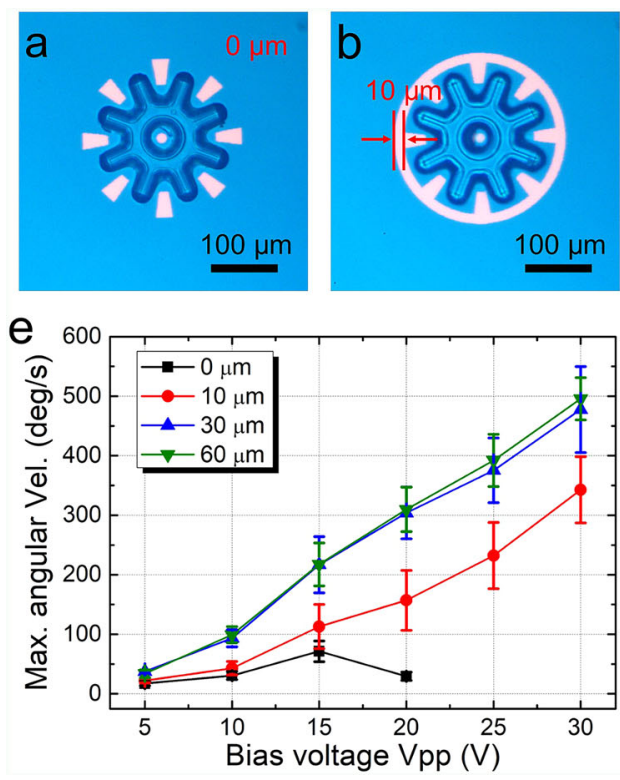
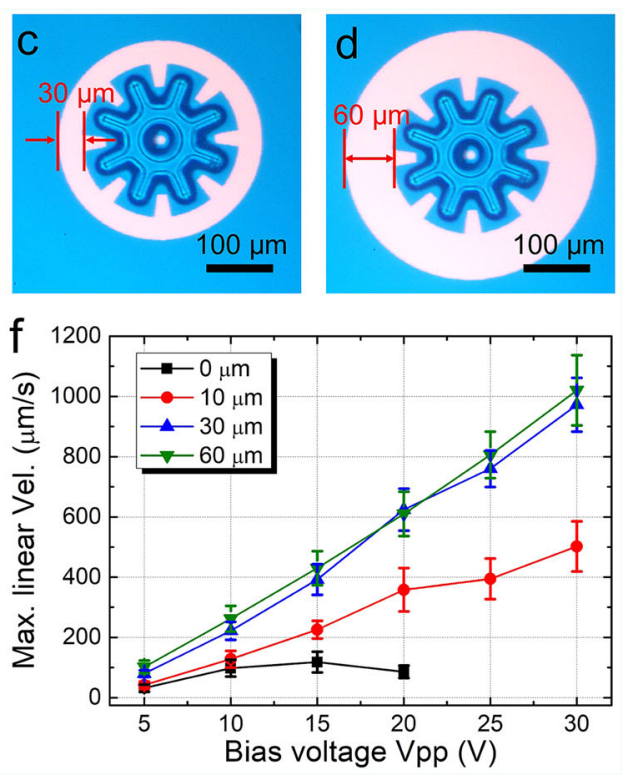

Fig. 2 Effect of optical ring-spanner geometry on micromotors. Microscope images of micro-gears controlled by optical ring spanners with ring thicknesses of $\mathbf{a} 0 \mu \mathrm{m}, \mathbf{b} 10 \mu \mathrm{m}, \mathbf{c} 30 \mu \mathrm{m}$, and $\mathbf{d} 60 \mu \mathrm{m}$. Plots of maximum e angular velocity of rotating micro-gears and $\mathbf{f}$ linear velocity of translating micro-gears as a function of OET bias voltage for optical ring spanners with ring thicknesses of $0 \mu \mathrm{m}$ (black squares), $10 \mu \mathrm{m}$ (red circles), $30 \mu \mathrm{m}$ (blue triangles), and $60 \mu \mathrm{m}$ (green inverted triangles). The frequency of the OET bias was set to $10 \mathrm{kHz}$. Error bars represent \pm 1 SD from five measurements for each condition.

accompanied by the observation that the failure modes differ for optical ring spanners with thin vs. thick rings. Micro-gears driven by spanners with thin rings were prone to a flipping failure mode (Supplementary Movie 4, clips 1 and 2), in which the micro-gear flipped up into the Z-dimension. In contrast, micro-gears driven by spanners with thick rings failed only by a stripping mode (Supplementary Movie 4, clip 3), akin to the mechanical stripping that is observed for a mechanical wrench that is rotated too rapidly. The flipping phenomenon, in particular, is unique, as it (unlike any other result discussed herein) is also occasionally observed in cases in which the bias voltage is applied, but the light pattern is not projected into the system (Supplementary Fig. 2). We describe this and other observations in more detail in
Supplementary Note 3; more study is merited to probe these interesting phenomena.

Micromotor impellers. With a unit component and its behavioral boundary conditions in hand, we turned to exploring the use of micro-gears as micromotors and micromachines. A common motif in the optical micromotor literature is the use of a spinning particle as an impeller, which generates hydrodynamic forces that influence the behavior of nearby particles ${ }^{10,12,34,39,40}$. The system described here behaves similarly-as shown in Supplementary Movie 5 and in Fig. 3a, b, a rotating micro-gear causes polystyrene microparticles in the vicinity to revolve around it. We 

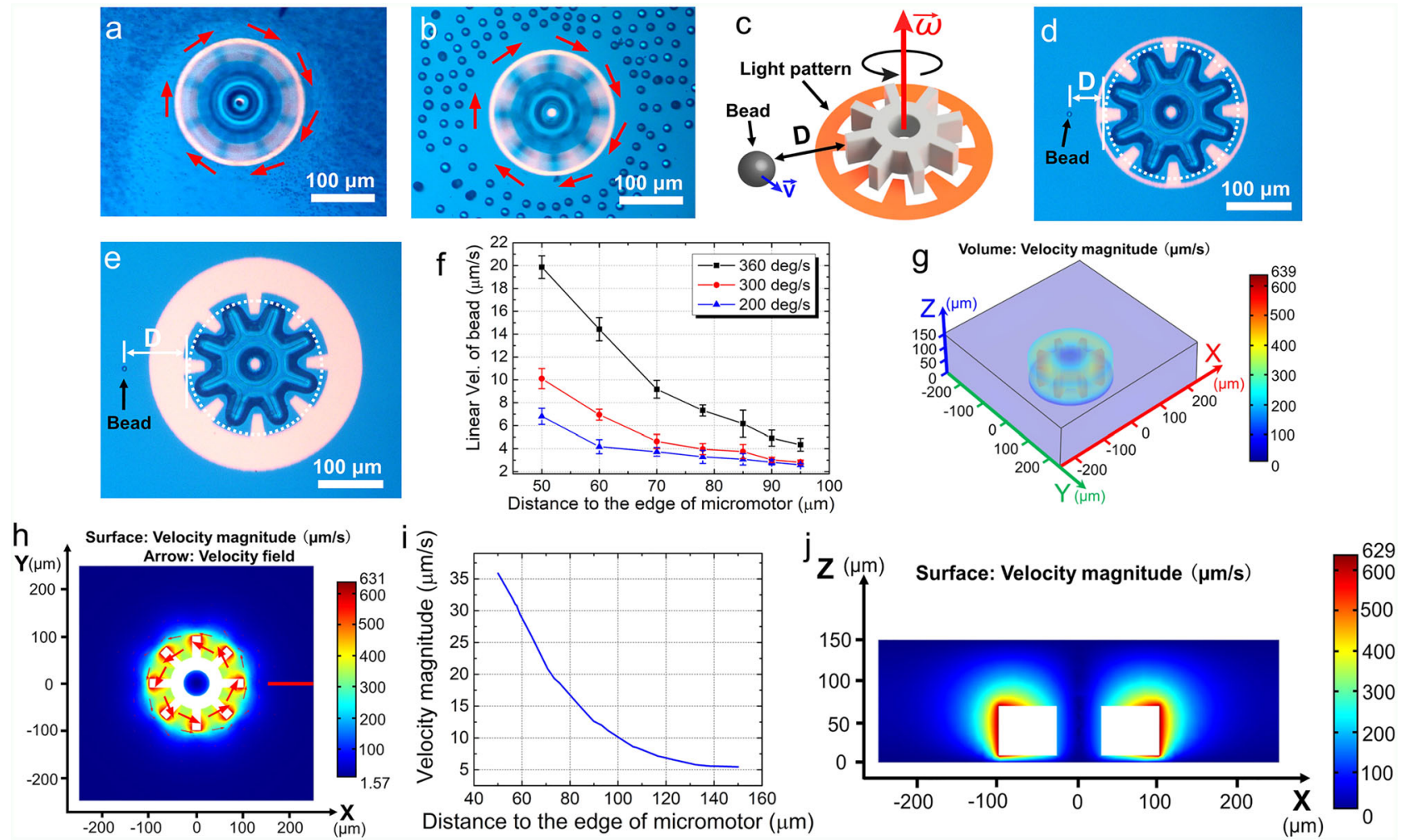

Fig. 3 Micromotor impellers. Frames from Supplementary Movie 5 of a micro-gear rotating at $360^{\circ} / \mathrm{s}$ in a suspension of a $1-\mu \mathrm{m}$-diameter and $\mathbf{b} 15-\mu \mathrm{m}-$ diameter polystyrene microbeads. Red arrows indicate the direction of rotation of the micro-gear and revolution of the particles. $\mathbf{c}$ Schematic of a rotating micro-gear (with angular velocity $\omega$ ) and its influence on a nearby particle (with linear velocity $v$ ), and distance $D$ between the two. Microscope images of a 7- $\mu \mathrm{m}$-diameter polystyrene microbead and a micro-gear with $\mathbf{d} D=50 \mu \mathrm{m}$ and $\mathbf{e} D=95 \mu \mathrm{m}$. $\mathbf{f}$ Linear velocities of 7- $\mu \mathrm{m}$-diameter beads revolving around rotating micro-gears as a function of $D$ for micro-gears rotated at $200 \%$ s (blue triangles), $300 \%$ s (red circles), and $360 \%$ s (black squares). Error bars represent \pm 1 SD from three measurements for each condition. 3D ( $\mathbf{g}), 2 \mathrm{D}(\mathbf{h}), 1 \mathrm{D}(\mathbf{i})$, and 2D (j) plots of numerical simulation of the velocity of flow (in $\mu \mathrm{m} / \mathrm{s}$ ) around a micro-gear rotating at $360 \% \mathrm{~s}$ after $2 \mathrm{~s}$ of rotation. Velocities in $(\mathbf{g}, \mathbf{h}$, and $\mathbf{j})$ are shown as a heat map from $0 \mu \mathrm{m} / \mathrm{s}$ (blue) to over $600 \mu \mathrm{m} / \mathrm{s}(\mathrm{red})$, and arrows in (h) are velocity vectors. $\mathbf{h}$ is an $X Y$ slice from $(\mathbf{g})$ at $Z=11 \mu \mathrm{m}$, $\mathbf{i}$ is an $X$ slice of (h) [denoted by the red line in (h)], and $\mathbf{j}$ is an $X Z$ slice from $(\mathbf{g})$ at $Y=0 \mu \mathrm{m}$ (the white boxes denote the position of the micro-gear).

decided to explore this phenomenon, and as illustrated in Fig. 3c, two variables were probed in these experiments-the distance $D$ from the particle to the micro-gear, and the angular velocity of the micro-gear. The former variable was controlled by varying the ring thickness in the optical ring spanner. As shown in Fig. 3d, e, the negative DEP force in this system establishes a minimum value for $D$, providing convenient means to control this parameter. The latter variable was controlled by rotating optical ring spanners and micro-gears at different angular velocities, as illustrated in Supplementary Movie 6 (clips 1 and 2). Importantly, control experiments were implemented to confirm that beads do not move when exposed to a rotating optical ring spanner only, with no micro-gear (Supplementary Movie 6, clip 3). Figure 3f shows the linear velocities observed for $7-\mu \mathrm{m}$-diameter polystyrene microbeads as they revolve around rotating micro-gears. As shown, the bead velocities have a strong dependence on both variables-decreased distance between particle and micro-gear, and increased angular velocity of micro-gear-both cause the beads to predictably revolve at higher linear velocities.

To better contextualize the experimental results, 3D numerical simulations of fluid flow around a rotating micro-gear were carried out in COMSOL Multiphysics. In this model, a simulated micro-gear was rotated continuously for $2 \mathrm{~s}$ to generate a stable hydrodynamic flow (see the detailed description of the model in Supplementary Note 4 and Supplementary Fig. 3). An animation of a 3D flow velocity distribution in time for a micro-gear rotated at $360 \% \mathrm{~s}$ can be seen in Supplementary Movie 7, and a still image from the animation at $t=2 \mathrm{~s}$ is shown in Fig. 3g. Two-dimensional slices can be generated from the simulation and visualized in Supplementary Movie 8 (XY slice for $Z=11 \mu \mathrm{m}$ ). A still image from this animation at $t=2 \mathrm{~s}$ is shown in Fig. 3h. A one-dimensional (X) slice can also be generated, as shown in Fig. 3i. The simulation indicates that there exists a localized field of vortex flow that drops off rapidly with increased distance from the micro-gear, which matches the experimental observations. In fact, the simulation (Fig. 3i) has a close agreement with the experiments (Fig. 3f) -for example, a 7- $\mu \mathrm{m}$ diameter polystyrene bead located $50 \mu \mathrm{m}$ from a micro-gear rotating at $360 \%$ has a linear velocity of around $20 \mu \mathrm{m} / \mathrm{s}$, while the simulated fluid velocity under the same conditions is $\sim 35 \mu \mathrm{m} / \mathrm{s}$. (Note that the bead's velocity should be reduced relative to that of the fluid, because of viscous drag.) Finally, flow velocity distribution in the vertical plane can be observed in Fig. 3j [XZ slice for $Y=0 \mu \mathrm{m}$ of Fig. 3g], and the torque in this simulation at $t=2 \mathrm{~s}$ was $1.6 \times 10^{-13} \mathrm{~N} \cdot \mathrm{m}$.

Micromachine 1: the touchless micro-feed-roller. The vast majority of micromotor reports in the literature ${ }^{10,11,14,34-38}$ (as well as the aforementioned OET microrobot ${ }^{56}$ ) describe the activity of a single micromotor (or what we reference as a unit component, as explored in Figs. 1-3). But we hypothesized that multicomponent micromachines relying on the coordinated behavior of more than one micromotor in tandem could provide additional functionalities, and we first tested the behavior of two micro-gears close together that are rotated in opposite directions. 
We call this a micro-feed-roller, by analogy to the pairs of cylinders that rotate to move the paper through a desktop printer. Unlike a conventional feed-roller, this system is touchless because the objects that are manipulated, such as the polystyrene beads in Supplementary Movie 9 and Fig. 4a, b, do not contact the rollers. In a hydrodynamic system such as this one, the physical extent of the control flow field is governed by the distance between the flow-actuating elements and the target objects. As shown in Fig. 4c, a particle translated by the touchless micro-feed-roller first accelerates and then decelerates, and the velocity of the particle is highest when it is in closest proximity to the microgears (in the midpoint of its path). This behavior is similar to what was reported previously for an OT-driven micromachine, which was found to be able to program particle position with nanometer spatial precision ${ }^{12}$.
The touchless micro-feed-roller is useful for moving particles across short distances; it can be modified to manipulate particles across large distances by projecting an OET pattern between the two rotating micro-gears and translating the entire system. We call this structure, shown in Supplementary Movie 10, clip 1 and Fig. 4d, an OET-bridged touchless micro-feed-roller, which combines OET manipulation with hydrodynamics. This system is particularly useful for manipulating particles that show weak responses to OET/DEP forces, such as mammalian cells. Specifically, when OET/DEP alone (with no hydrodynamics) is used to translate a B-16 cell, as in Supplementary Movie 10, clip 2 and Fig. 4e, the maximum linear velocity is slow, as viscous drag readily overwhelms the weak OET/DEP forces at high velocities. But when hydrodynamics is added to the system, as in the OET-bridged touchless micro-feed-roller, cells can be manipulated more rapidly
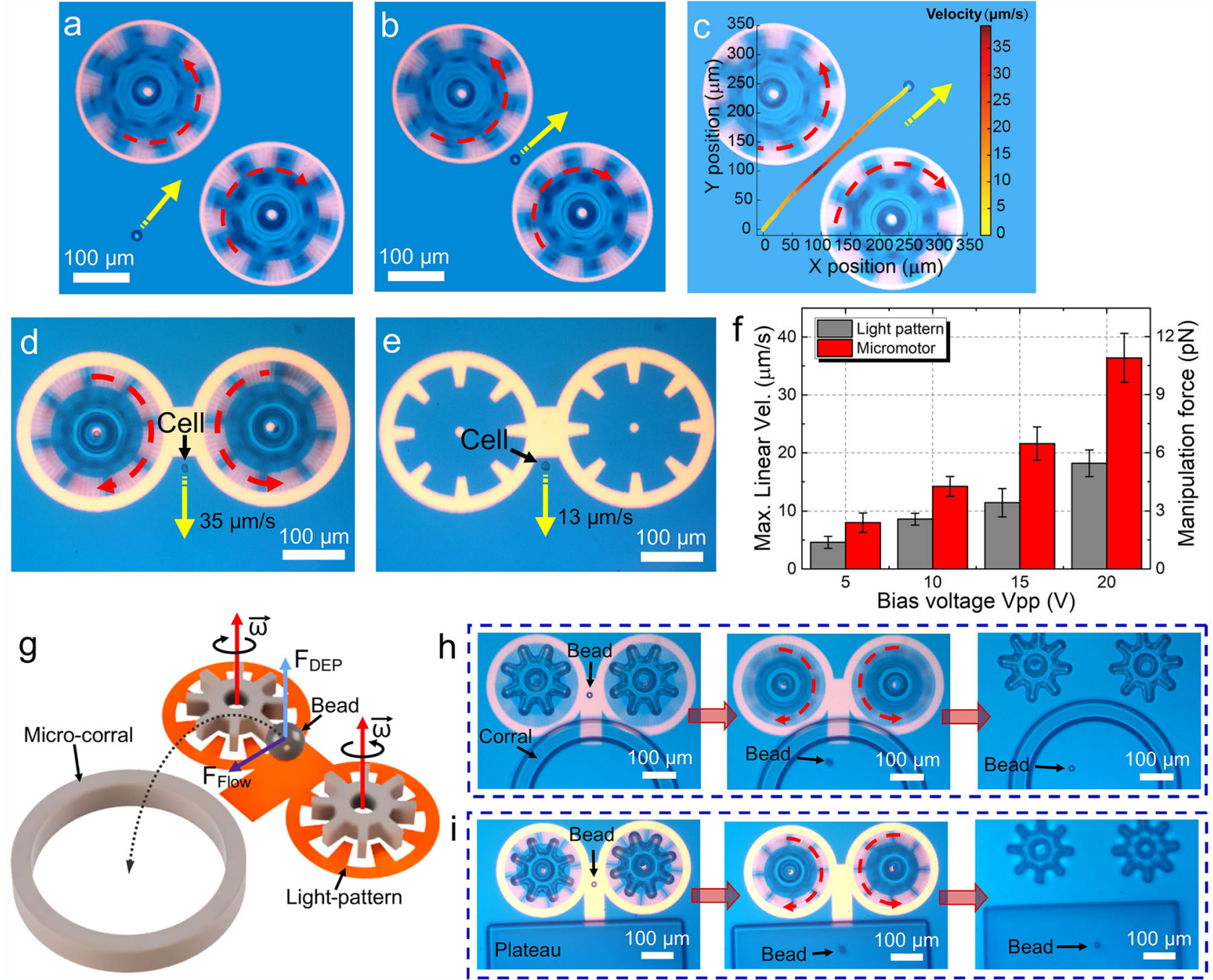

Fig. 4 Micromachine 1: the touchless micro-feed-roller. Frames from Supplementary Movie 9 at time a $t=0 \mathrm{~s}, \mathbf{b} 12 \mathrm{~s}$, and $\mathbf{c} 27 \mathrm{~s}$, depicting the position of a 20- $\mu \mathrm{m}$-diameter polystyrene microbead propelled (with the direction indicated by yellow arrows) by a touchless micro-feed-roller. A plot of Cartesian coordinates of the bead from $t=0$ to $27 \mathrm{~s}$ is superimposed on the image in (c), with bead velocity indicated in a heat map (yellow =stationary, $\mathrm{red}=40 \mu \mathrm{m} / \mathrm{s}$ ). Frames from Supplementary Movie 10 depicting the translation of a B-16 cell translated at $\mathbf{d} 35 \mu \mathrm{m} / \mathrm{s}$ by an OET-bridged touchless microfeed-roller and $\mathbf{e} 13 \mu \mathrm{m} / \mathrm{s}$ by the light pattern alone. $\mathbf{f}$ Plot of maximum translation velocity (left axis) and corresponding force (right axis) as a function of bias voltage for the manipulation of B-16 cells by OET-bridged touchless micro-feed-roller (red) and light pattern (OET) alone (gray). Error bars represent \pm 1 SD from five measurements for each condition. $\mathbf{g}$ Schematic of an OET-bridged touchless micro-feed-roller used to cause a microbead to hop into a circular micro-corral. Frames from Supplementary Movie 11 illustrating the use of an OET-bridged touchless micro-feed-roller to cause a 15- $\mu$ m-diameter microbead to (h) hop into a circular micro-corral and (i) hop onto a micro-plateau. Dashed red lines with arrowheads indicate the direction of rotation of micromotors in relevant panels. 
and with greater force (calculated from velocity via Stokes' law ${ }^{48,57}$ ), as illustrated in Fig. $4 \mathrm{f}$ for a range of different conditions.

Most importantly, in exploring the behavior of the OET-bridged touchless micro-feed-roller, we discovered a unique property that extends the functionality beyond two dimensions. Specifically, as illustrated in Fig. $4 \mathrm{~g}$, by carefully tuning the $Z$-axis DEP force ${ }^{58,59}$ from the OET-bridge and the $X Y$-axis hydrodynamic force of the micro-feed-roller, objects can be made to controllably hop in three dimensions. (Note that previous demonstrations of similar systems have been exclusively $2 \mathrm{D}^{12}$.) To probe this capacity, SU- 8 microstructures were formed to serve as $3 \mathrm{D}$ obstacles (Supplementary Fig. 4). As shown in Supplementary Movie 11, clip 1 and Supplementary Fig. 5a, b, XY-manipulation using a simple OET doughnut-shaped light pattern causes microbeads to remain in one plane such that they crash into the edges of micro-structures, becoming dislodged. In contrast, as shown in Supplementary Movie 11, clip 2, the XYZ-forces generated by the OET-bridged touchless micro-feed-roller reproducibly cause particles to hop over (Supplementary Fig. 5c), into (Fig. 4h and Supplementary Fig. 5d) or onto (Fig. 4i) various kinds of micro-structures. As described in Supplementary Note 5, the vertical displacement (in the $Z$-dimension) at the apogee of each hop was estimated to be $\sim 135 \mu \mathrm{m}$. Finally, as shown in Supplementary Movie 11, clip 3, when the $Z$-axis OET/DEP force is applied without the hydrodynamic force of the micro-feed-roller (i.e., with no operation of the micromotor), the bead hops, but does so uncontrollably (and cannot be propelled over, onto, or into a micro-structure)-the hydrodynamic force is necessary to control and predict the position of the bead. We are unaware of any other micromachine that affords this type of 3D control over secondary particles, which is analogous to a "basketball shot" or a "missile launch." This capacity should allow for great potential to construct new variations of three-dimensional microstructures for a wide range of applications.

Micromachine 2: the micro-gear train. As a next step, we decided to investigate whether the multicomponent micromachines introduced here can provide a mechanical advantage. Specifically, we explored the possibility of driving the rotation of one or more micro-gears with optical ring spanners and then using the torque generated by this active process to drive the rotation of one or more passive micro-gears in a micro-gear train. We are aware of only a single previous report ${ }^{16}$ of a similar system (driven by OT), which was purely descriptive, with no details about gear size, rotation velocity, or mechanical advantage. For the current system, Supplementary Movie 12 illustrates the behavior of micro-gear trains comprising two (Fig. 5a) or four (Fig. 5b) micro-gears. A single active micro-gear generates a finite torque (e.g., we estimate $1.6 \times 10^{-13} \mathrm{~N} \cdot \mathrm{m}$ for a micro-gear rotating at $360^{\circ} / \mathrm{s}$ ) that can be used to drive a micro-gear train. As expected, each additional passive micro-gear increases the viscous stress and friction for the micromachine, reducing the maximum angular velocity of the system-this effect was measured and is shown in Fig. 5c. These limits can be overcome by
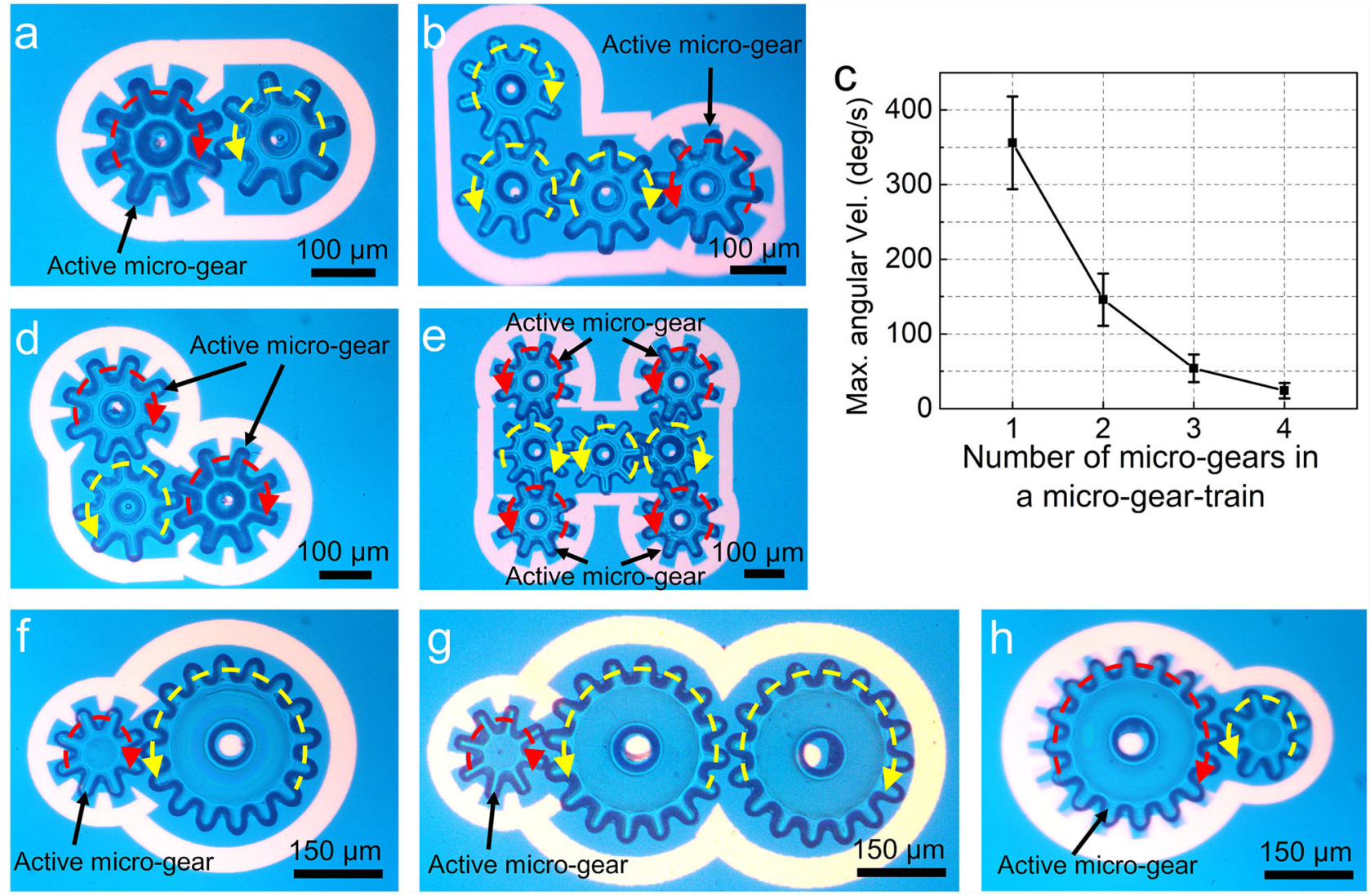

Fig. 5 Micromachine 2: the micro-gear train. Frames from Supplementary Movie 12 illustrating micro-gear-trains driven by one active micro-gear comprising $\mathbf{a}$ two and $\mathbf{b}$ four micro-gears. c Plot of maximum angular velocity as a function of the number of components in a micro-gear-trains with one active micro-gear driven by a $20 V_{p p}$ OET bias voltage at $10 \mathrm{kHz}$. Error bars represent $\pm 1 \mathrm{SD}$ from five measurements for each type of micro-gear-train (assembled from different components). Frames from Supplementary Movie 13 illustrating micro-gear-trains driven by d two active micro-gears (three total) and $\mathbf{e}$ four active micro-gears (seven total). $\mathbf{f}-\mathbf{h}$ Frames from Supplementary Movie 14 illustrating micro-gear-trains formed from small (150 $\mu \mathrm{m}$ diameter) and large micro-gears ( $300 \mu \mathrm{m}$ diameter). In (f, $\mathbf{g})$, the small micro-gear drives the system; in (h), the large micro-gear drives the system. In all images, red and yellow dashed lines with arrowheads represent the rotation directions of the active and passive micro-gears, respectively. 
using multiple active micro-gears to drive a gear train, as illustrated by Supplementary Movie 11 for three (Fig. 5d) and seven (Fig. 5e) micro-gears.

As described in Supplementary Note 6, the micromachines in Supplementary Movies 12 and 13 and Fig. 5a, b, d, e, which are formed from micro-gears with the same size, have a mechanical advantage $\mathrm{MA}=1$. To test the capacity to form torque multipliers with $M A>1$ or speed multipliers with $\mathrm{MA}<1$, micro-gears with varying sizes (Supplementary Fig. 6) were formed and assembled into micro-gear trains. Supplementary Movie 14, clips 1 and 2 (Fig. 5f, g) depict micromachines with $\mathrm{MA}=2$, which amplify the input torque two-fold. Supplementary Movie 14, clip 3 (Fig. 5h) depicts a micromachine with $\mathrm{MA}=0.5$, which amplifies the input angular velocity by a factor of 2 . Specifically, in this example the active gear was rotated at $100 \%$, causing the passive gear to rotate at $200 \%$ s (measured frame to frame in Supplementary Movie 14). This behavior was reproducible across multiple micro-gear trains (comprising different combinations of large and small gears), suggesting exciting possibilities for more complex light-driven micromachines in the future.

Micromachine 3: the micro-rack-and-pinion. Finally, we turned our attention to a different type of multicomponent micromachine-a micro-rack-and-pinion system (Fig. 6a) that transforms rotary into linear motion. In this system, a standard microgear (operated as described above) serves as the pinion, while a new component-a linear gear with commensurate dimensions (Supplementary Note 7 and Supplementary Fig. 7a, b) - serves as the rack. As illustrated in Supplementary Movie 15, clips 1 and 2, upon engaging the two components, rotating an optical ring spanner counterclockwise (Fig. 6b) or clockwise (Fig. 6c) causes the (active) pinion to rotate, which causes the (passive) rack to move linearly to the left or to the right, respectively.

We can foresee myriad applications of the micro-rack-andpinion system, which is (to our knowledge) entirely unique in the micromotor/machine literature. As an example, a custom microfluidic structure featuring three parallel microchannels (Fig. 6d and Supplementary Fig. $7 \mathrm{c}-\mathrm{h}$ ) was designed and integrated into the OET chamber to evaluate the potential of using micro-rack-and-pinion systems to serve as a variable control valve. Specifically, as illustrated in Supplementary Movie 15, clips 3-6, when oriented appropriately, rotating pinions on either side of the microfluidic structure causes micro-racks to penetrate the microchannel junction (Fig. 6e, f), impeding the flow within them. By controlling the two micro-rack-and-pinion structures in tandem, the system can be configured to isolate the flow into any of the three channels in the system when driven by flow rates of up to $5 \mu \mathrm{L} / \mathrm{min}$ (Fig. $6 \mathrm{~g}-\mathrm{i}$ ). As an example, the valve state can be used to control the flow of a suspension of microparticles, as illustrated in Supplementary Movie 16 (Fig. 6j, k). We note that there have been many useful types of mechanical microfluidic valves reported over the years ${ }^{60-65}$, but the system illustrated in Fig. 6 is the first example that we are aware of that is realized using multicomponent micromachines on-chip. Most importantly, the valves shown here are just an example. We propose that the general principles illustrated in Figs. 3-6, which include 3D particle control, torque/speed multiplication, and mechanical conversion of rotary/linear motion, may eventually be useful for applications that have not yet been imagined in hydrodynamics, microrobotics, microfluidics, and beyond.

In sum, we have introduced a new member of the micromotor family-gear-shaped components that can be rotated and
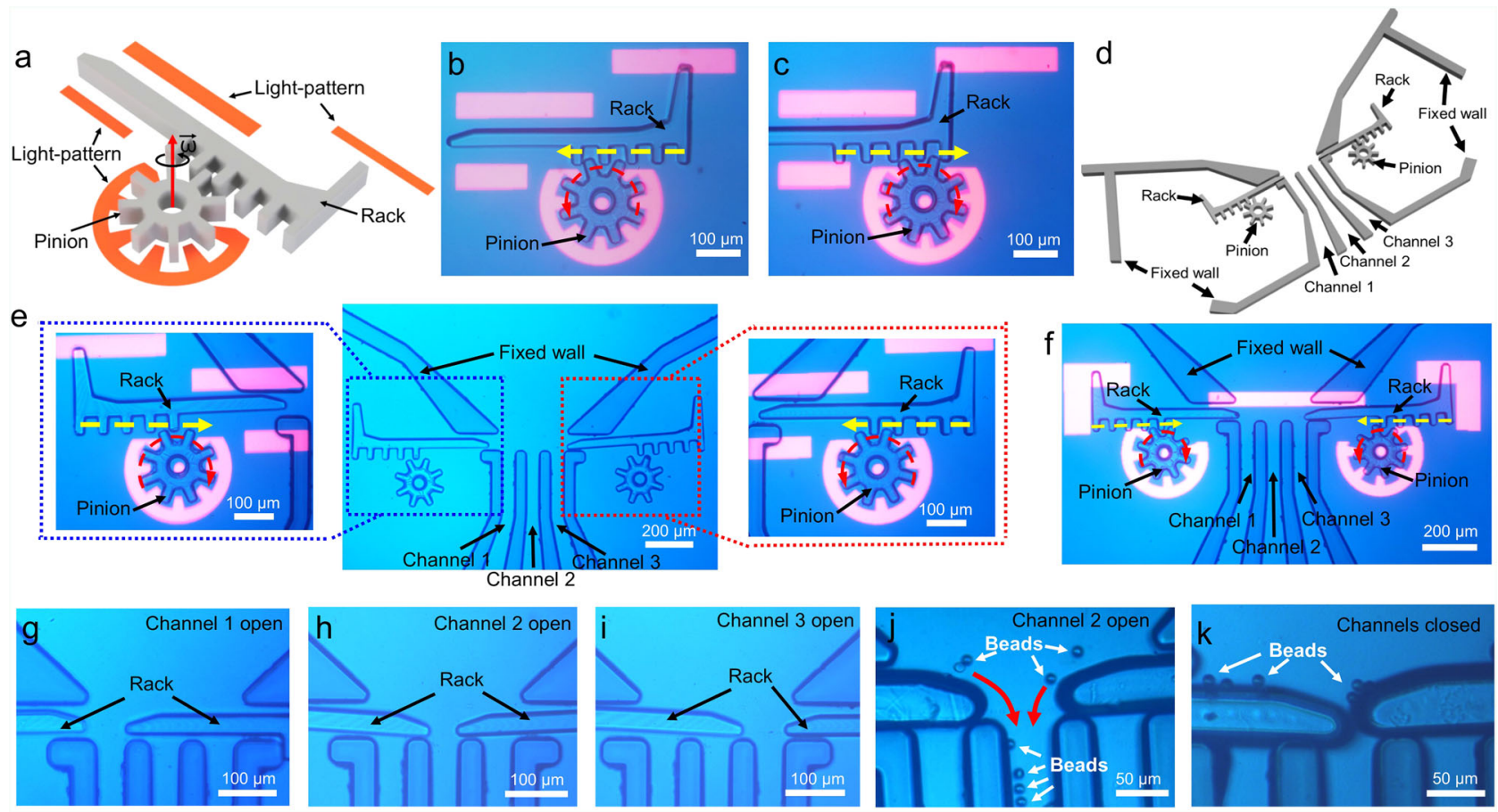

Fig. 6 Micromachine 3: the micro-rack-and-pinion. a Schematic of a micro-rack-and-pinion system. b, c Frames from Supplementary Movie 15 illustrating a micro-rack-and-pinion system in operation, in which rotating the pinion counterclockwise or clockwise can make the rack translate to the left or the right, respectively (with the direction indicated by yellow dashed arrows). d Schematic of a permanent microchannel structure designed to interface with microrack-and-pinion systems. e Microscope images of a microfluidic valve formed from two micro-rack-and-pinion systems that can be individually controlled. f-i Frames from Supplementary Movie 15 illustrating how two rack and pinion structures can work together to choke and isolate flow in the microchannels. j, k Frames from Supplementary Movie 16 illustrating the use of micro-rack-and-pinion structures as valves to control the movement of 10- $\mu m$-diameter microbeads in microfluidic channels. In $(\mathbf{b}, \mathbf{c}, \mathbf{e}, \mathbf{f})$, red and yellow dashed lines with arrowheads represent the motion of the (active) pinions and the (passive) racks, respectively. In (j), red lines with arrowheads represent the moving direction of the microbeads. 
translated by light-driven dielectrophoresis. Like micromotors described previously, the micro-gear components described here can be made to serve as impellers to generate localized hydrodynamic forces to control the motion of micro-objects in their vicinity. In an advance relative to the state of the art, microgears can be made to work together as micromachines, allowing for 3D particle control, mechanical advantage, and microfluidic valving behaviors to be realized. We propose that this represent an important step forward for the microsystems community, opening the door to ever more complex and useful micromachines in the future.

\section{Methods}

OET system and characterization. The OET setup comprises a DMD-based pattern illuminator (Mightex Polygon 1000G, $625 \mathrm{~nm} 1100 \mathrm{~mW}$ LED source) interfaced with an upright optical microscope (Leica DM 2000 microscope integrated with Märzhäuser Scan Plus $100 \times 100$ motorized stage), using a $\times 10$ or $\times 5$ objective. The optical power densities of light patterns projected through the microscope were measured using a Thorlabs PM16-130 power meter to be 0.44 and $0.12 \mathrm{~W} / \mathrm{cm}^{2}$ for the $\times 10$ objective and $\times 5$ objective, respectively (and no opticalinduced heating was observed for these conditions). Finally, the resolution of optical patterns (defined as the lateral distance between two distinguishable optical points generated by adjacent DMD pixels) formed in this system are 0.8 and $1.5 \mu \mathrm{m}$ for the $\times 10$ objective and $\times 5$ objective, respectively. OET devices were similar to those reported previously $49,51,56$, consisting of a $20 \mu \mathrm{L}$ fluidic chamber sandwiched between two glass plates separated by a $150 \mu \mathrm{m}$ spacer. The plates are coated with a thin layer of indium tin oxide (ITO), and the bottom plate is coated with an additional layer of a-Si:H (see Fig. $1 d$ ). AC potentials $\left(5-30 V_{\mathrm{pp}}, 10-30 \mathrm{kHz}\right.$, sine waves) used to drive the OET device were supplied by a function generator (Agilent 33220A) and an amplifier (Thurlby Thandor Instrument WA31). At these frequencies, the sign of the real part of the Clasius-Mossotti factor is negative for the particles (photoresist micromotors, polystyrene beads, and biological cells) and liquid media (aqueous solutions with conductivity $<30 \mathrm{mS} / \mathrm{m}$ ) used here. When not specified otherwise, the OET bias was $30 V_{\mathrm{pp}}$ at $10 \mathrm{kHz}$. In this frequency regime, $\mathrm{DEP}$ is the dominant force relative to $\mathrm{AC}$ electroosmosis ${ }^{66}$, and was used here because of empirical observation of high-fidelity micromotor movement in response to light pattern positions. SEM images were collected using an environmental SEM (QUANTA FEG 250 ESEM), with a pressure of $130 \mathrm{~Pa}$ and an electron beam with $10 \mathrm{keV}$ energy and $3 \mathrm{~nm}$ spot size. 3D profiles were measured by an optical profilometer (Bruker Contour GT-K).

Microfabrication. Micro-gears (including circular and linear/rack-style gears) were formed from SU-8 2050 (MicroChem, USA) at the University of Toronto's Centre for Research and Applications in Fluidic Technologies (CRAFT) cleanroom facility using methods similar to those reported previously ${ }^{56}$. Briefly, $1 \mathrm{~mL}$ Omnicoat (MicroChem, USA) was spin-coated on top of a 4 in. silicon wafer (2000 r.p.m. for $30 \mathrm{~s}$ ), followed by soft baking at $200^{\circ} \mathrm{C}$ for $1 \mathrm{~min}$. After cooling the silicon wafer to room temperature, $4 \mathrm{~mL}$ SU-8 2050 was spin-coated at 2500 r.p.m. (for $\sim 60-\mu \mathrm{m}$ thick micro-gears) or 3500 r.p.m. (for $\sim 30-\mu \mathrm{m}$-thick micro-gears) for $30 \mathrm{~s}$ atop the silicon substrate, followed by soft baking at $65^{\circ} \mathrm{C}$ for $3 \mathrm{~min}$ and $95^{\circ} \mathrm{C}$ for $8 \mathrm{~min}$. Next, a mask aligner (OAI model 30 ) was used to illuminate the substrates (exposure energy: $9 \mathrm{~mJ} / \mathrm{cm}^{2}$ for $20 \mathrm{~s}$ ) through a photomask to selectively photocrosslink the SU-8. After postexposure baking $\left(65^{\circ} \mathrm{C}\right.$ for $2 \mathrm{~min}$ and $95^{\circ} \mathrm{C}$ for $7 \mathrm{~min}$ ) and developing in SU-8 developer $(8 \mathrm{~min})$, the substrates were air-dried with pressurized nitrogen. The substrates were then immersed in Remover PG (Microchem, USA) to dissolve the Omnicoat ( 3 min under gentle agitation) and to lift the photo-cured SU-8 micro-gears into suspension. The suspension was collected into a $15 \mathrm{~mL}$ tube and centrifuged at $14,500 \times g$ for $30 \mathrm{~s}$, the supernatant was removed, and the micro-gears were resuspended in bead medium [deionized (DI) water containing $0.05 \%$ volume ratio of Tween-20, P9416, Sigma] or sucrose medium [DI water, $9 \mathrm{wt} \%$ sucrose, $0.3 \mathrm{wt} \%$ D-glucose, $1.25 \%(\mathrm{v} / \mathrm{v})$ phosphatebuffered saline (PBS)]. The centrifuge/resuspension process was repeated three times, after which the suspensions were stored at room temperature until use. Immediately prior to use, each micro-gear suspension was gently vortexed and an aliquot $(15 \mu \mathrm{L})$ was loaded into the chamber of an OET device, followed by loading a second aliquot $(5 \mu \mathrm{L})$ of bead or cell suspension.

Sample preparation. Suspensions of polystyrene microparticles of different diameters $\left(1,3,7\right.$, and $15 \mu \mathrm{m}$, Polysciences) were formed at $0.1-1 \times 10^{7}$ particles $/ \mathrm{mL}$ in bead medium (with a conductivity of $5.0 \mathrm{mS} / \mathrm{m}$ ) prior to loading into OET devices. B-16 cells were cultured in DMEM (Life Technologies) with $10 \%$ (v/v) fetal bovine serum (Gibco), and $1 \%(\mathrm{v} / \mathrm{v})$ penicillin and streptomycin (Invitrogen) in cell culture flasks in a humidified incubator with $5 \%(\mathrm{v} / \mathrm{v}) \mathrm{CO}_{2} /$ air at $37^{\circ} \mathrm{C}$. Prior to experiments, cells were washed twice in PBS (Life Technologies), passaged in $0.25 \%$ Trypsin-EDTA (Life Technologies), and centrifuged and resuspended at $0.1-1 \times 10^{6}$ cells $/ \mathrm{mL}$ in sucrose medium (with a conductivity of $22.1 \mathrm{mS} / \mathrm{m}$ ).
Finally, suspensions were filtered through a $40-\mu \mathrm{m}$ cell strainer (Falcon) prior to loading into OET devices.

\section{Data availability}

The data generated in this study are provided in the Supplementary information/Source data file. Source data are provided with this paper.

Received: 22 February 2021; Accepted: 5 August 2021; Published online: 09 September 2021

\section{References}

1. Wang, J. \& Gao, W. Nano/microscale motors: biomedical opportunities and challenges. ACS Nano. 6, 5745-5751 (2012).

2. Alapan, Y., Yigit, B., Beker, O., Demirörs, A. F. \& Sitti, M. Shape-encoded dynamic assembly of mobile micromachines. Nat. Mater. 18, 1244-1251 (2019).

3. de Ávila, B. E.-F., Gao, W., Karshalev, E., Zhang, L. \& Wang, J. Cell-like micromotors. Acc. Chem. Res. 51, 1901-1910 (2018).

4. Hu, W., Lum, G. Z., Mastrangeli, M. \& Sitti, M. Small-scale soft-bodied robot with multimodal locomotion. Nature 554, 81-85 (2018).

5. Li, A. et al. Programmable droplet manipulation by a magnetic-actuated robot. Sci. Adv. 6, eaay5808 (2020)

6. Wang, W. et al. Acoustic propulsion of nanorod motors inside living cells. Angew. Chem. Int. Ed. Engl. 53, 3201-3204 (2014).

7. Ren, L. et al. Rheotaxis of bimetallic micromotors driven by chemical-acoustic hybrid power. ACS Nano 11, 10591-10598 (2017).

8. Wyatt Shields, C. IV et al. Supercolloidal spinners: complex active particles for electrically powered and switchable rotation. Adv. Funct. Mater. 28, 1803465 (2018)

9. Xu, X., Liu, C., Kim, K. \& Fan, D. L. Electric-driven rotation of silicon nanowires and silicon nanowire motors. Adv. Funct. Mater. 24, 4843-4850 (2014).

10. $\mathrm{Wu}, \mathrm{T}$. et al. A photon-driven micromotor can direct nerve fibre growth. Nat Photon. 6, 62-67 (2012)

11. Metzger, N. K., Mazilu, M., Kelemen, L., Ormos, P. \& Dholakia, K. Observation and simulation of an optically driven micromotor. J. Opt. 13, 044018 (2011).

12. Būtaité, U. G. et al. Indirect optical trapping using light driven micro-rotors for reconfigurable hydrodynamic manipulation. Nat. Commun. 10, 1215 (2019).

13. Neale, S. L., MacDonald, M. P., Dholakia, K. \& Krauss, T. F. All-optical control of microfluidic components using form birefringence. Nat. Mater. 4 , 530-533 (2005).

14. Bianchi, S., Vizsnyiczai, G., Ferretti, S., Maggi, C. \& Leonardo, R. D. An optical reaction micro-turbine. Nat. Commun. 9, 4476 (2018).

15. Maggi, C., Saglimbeni, F., Dipalo, M., Angelis, F. D. \& Leonardo, R. D. Micromotors with asymmetric shape that efficiently convert light into work by thermocapillary effects. Nat. Commun. 6, 7855 (2015).

16. Galajda, P. \& Ormos, P. Complex micromachines produced and driven by light. Appl. Phys. Lett. 78, 249-251 (2001).

17. Glückstad, J. \& Palima, D. Light Robotics-Structure-Mediated Nanobiophotonics (Elsevier, 2017).

18. Mohanty, S. Optically-actuated translational and rotational motion at the microscale for microfluidic manipulation and characterization. Lab Chip 12, 3624-3636 (2012).

19. Palima, D. \& Glückstad, J. Gearing up for optical microrobotics: micromanipulation and actuation of synthetic microstructures by optical forces. Laser Photon. Rev. 7, 478-494 (2013).

20. Bunea, A. I. \& Glückstad, J. Strategies for optical trapping in biological samples: aiming at microrobotic surgeons. Laser Photon. Rev. 13, 1800227 (2019).

21. Zeng, $H$. et al. High-resolution 3D direct laser writing for liquid-crystalline elastomer microstructures. Adv. Mater. 26, 2319-2322 (2014).

22. Zeng, H., Wasylczyk, P., Wiersma, D. S. \& Priimagi, A. Light robots: bridging the gap between microrobotics and photomechanics in soft materials. Adv. Mater. 30, 1703554 (2018).

23. Palagi, S. et al. Structured light enables biomimetic swimming and versatile locomotion of photoresponsive soft microrobots. Nat. Mater. 15, 647-653 (2016).

24. Wani, O. M., Zeng, H. \& Priimagi, A. A light-driven artificial flytrap. Nat. Commun. 8, 15546 (2017).

25. Wang, X. Q. et al. Somatosensory, light-driven, thin-film robots capable of integrated perception and motility. Adv. Mater. 32, 2000351 (2020)

26. Lv, J. et al. Photocontrol of fluid slugs in liquid crystal polymer microactuators. Nature 537, 179-184 (2016)

27. Xu, L., Mou, F., Gong, H., Luo, M. \& Guan, J. Light-driven micro/nanomotors: from fundamentals to applications. Chem. Soc. Rev. 46, 6905-6926 (2017). 
28. Villa, K. \& Pumera, M. Fuel-free light-driven micro/nanomachines: artificial active matter mimicking nature. Chem. Soc. Rev. 48, 4966-4978 (2019).

29. Sridhar, V., Park, B. W. \& Sitti, M. Light-driven Janus hollow mesoporous $\mathrm{TiO}_{2}-\mathrm{Au}$ microswimmers. Adv. Funct. Mater. 28, 1704902 (2018).

30. Palagi, S., Singh, D. P. \& Fischer, P. Light-controlled micromotors and soft microrobots. Adv. Opt. Mater. 7, 1900370 (2019).

31. Aubret, A., Youssef, M., Sacanna, S. \& Palacci, J. Targeted assembly and synchronization of self-spinning microgears. Nat. Phys. 14, 1114-1118 (2018).

32. Phillips, D. B. et al. Shape-induced force fields in optical trapping. Nat. Photon. 8, 400-405 (2014).

33. Villangca, M. J., Palima, D., Bañas, A. R. \& Glückstad, J. Light-driven microtool equipped with a syringe function. Light Sci. Appl. 5, e16148 (2016).

34. Asavei, T. et al. Optically trapped and driven paddle-wheel. N. J. Phys. 15, 063016 (2013)

35. Köhler, J., Ksouri, S. I., Esen, C. \& Ostendorf, A. Optical screw-wrench for microassembly. Microsyst. Nanoeng. 3, 16083 (2017)

36. Friese, M. E., Rubinsztein-Dunlop, H., Gold, J., Hagberg, P. \& Hanstorp, D Optically driven micromachine elements. Appl. Phys. Lett. 78, 547-549 (2001).

37. Lin, X. F. et al. A light-driven turbine-like micro-rotor and study on its light-tomechanical power conversion efficiency. Appl. Phys. Lett. 101, 113901 (2012).

38. Maruo, S., Takaura, A. \& Saito, Y. Optically driven micropump with a twin spiral microrotor. Opt. Exp. 17, 18525-18532 (2009).

39. Leach, J., Mushfique, H., Leonardo, R., Padgett, M. \& Cooper, J. An optically driven pump for microfluidics. Lab Chip 6, 735-739 (2006).

40. Zou, X., Zheng, Q., Wu, D. \& Lei, H. Controllable cellular micromotors based on optical tweezers. Adv. Funct. Mater. 30, 2002081 (2020).

41. Woerdemann, M., Alpmann, C., Esseling, M. \& Denz, C. Advanced optical trapping by complex beam shaping. Laser Photon. Rev. 7, 839-854 (2013).

42. Wu, M. C. Optoelectronic tweezers. Nat. Photon. 5, 322-324 (2011).

43. Chiou, P. Y., Ohta, A. T. \& Wu, M. C. Massively parallel manipulation of single cells and microparticles using optical images. Nature 436, 370-375 (2005).

44. Hwang, H. \& Park, J. K. Optoelectrofluidic platforms for chemistry and biology. Lab Chip 11, 33-47 (2011).

45. Liang, W., Liu, L., Zhang, H., Wang, Y. \& Li, W. J. Optoelectrokinetics-based microfluidic platform for bioapplications: a review of recent advances. Biomicrofluidics 13, 051502 (2019).

46. Pethig, R. Review Article-dielectrophoresis: status of the theory, technology and applications. Biomicrofluidics 4, 022811 (2010).

47. Zhang, Y. et al. Detection and isolation of free cancer cells from ascites and peritoneal lavages using optically induced electrokinetics (OEK). Sci. Adv. 6, eaba9628 (2020)

48. Yang, S. M. et al. Dynamic manipulation and patterning of microparticles and cells by using TiOPc-based optoelectronic dielectrophoresis. Opt. Lett. 35, 1959-1961 (2010).

49. Zhang, S. et al. Patterned optoelectronic tweezers: a new scheme for selecting, moving, and storing dielectric particles and cells. Small 14, 1803342 (2018).

50. Yang, W., Yu, H., Li, G., Wang, Y. \& Liu, L. High-throughput fabrication and modular assembly of 3D heterogeneous microscale tissues. Small 13, 1602769 (2017).

51. Zhang, S. et al. Assembly of topographical micropatterns with optoelectronic tweezers. Adv. Opt. Mater. 7, 1900669 (2019).

52. Zhang, S. et al. Manufacturing with light-micro-assembly of opto-electronic microstructures. Opt. Express 25, 28838-28850 (2017).

53. Zhang, S. et al. Use of optoelectronic tweezers in manufacturing-accurate solder bead positioning. Appl. Phys. Lett. 109, 221110 (2016).

54. Juvert, J. et al. Micromanipulation of InP lasers with optoelectronic tweezers for integration on a photonic platform. Opt. Express 24, 18163-18175 (2016).

55. Zhang, S., Juvert, J., Cooper, J. M. \& Neale, S. L. Manipulating and assembling metallic beads with optoelectronic tweezers. Sci. Rep. 6, 32840 (2016).

56. Zhang, S. et al. The optoelectronic microrobot: a versatile toolbox for micromanipulation. Proc. Natl Acad. Sci. USA 116, 14823-14828 (2019).

57. Zhang, S. et al. Size-scaling effects for microparticles and cells manipulated by optoelectronic tweezers. Opt. Lett. 44, 4171-4174 (2019).

58. Zhang, S. et al. Escape from an optoelectronic tweezer trap: experimental results and simulations. Opt. Exp. 26, 5300-5309 (2018).

59. Zhao, Y., Lai, H. S., Zhang, G., Lee, G. B. \& Li, W. J. Rapid determination of cell mass and density using digitally controlled electric field in a microfluidic chip. Lab Chip 14, 4426-4434 (2014)

60. Unger, M. A., Chou, H. P., Thorsen, T., Scherer, A. \& Quake, S. R. Monolithic microfabricated valves and pumps by multilayer soft lithography. Science 288, 113-116 (2000).

61. Hasselbrink, E. F., Shepodd, T. J. \& Rehm, J. E. High-pressure microfluidic control in lab-on-a-chip devices using mobile polymer monoliths. Anal. Chem. 74, 4913-4918 (2002).
62. Gu, W., Zhu, X., Futai, N., Cho, B. S. \& Takayama, S. Computerized microfluidic cell culture using elastomeric channels and Braille displays. Proc. Natl Acad. Sci. USA 101, 15861-15866 (2004).

63. Grover, W. H., Ivester, R. H., Jensen, E. C. \& Mathies, R. A. Development and multiplexed control of latching pneumatic valves using microfluidic logical structures. Lab Chip 6, 623-631 (2006).

64. Gong, H., Woolley, A. T. \& Nordin, G. P. High density 3D printed microfluidic valves, pumps, and multiplexers. Lab Chip 16, 2450-2458 (2016)

65. Arango, Y., Temiz, Y., Gökçe, O. \& Delamarche, E. Electro-actuated valves and self-vented channels enable programmable flow control and monitoring in capillary-driven microfluidics. Sci. Adv. 6, eaay8305 (2020).

66. Valley, J. K., Jamshidi, A., Ohta, A. T., Hsu, H. Y. \& Wu, M. C. Operational regimes and physics present in optoelectronic tweezers. J. Microelectromech. Syst. 17, 342-350 (2008).

\section{Acknowledgements}

This research was supported by the Natural Sciences and Engineering Research Counci of Canada (Grant Nos. RGPIN 2019-04867, CREATE 482073-16, ALLRP 548593-19, and RTI-2019-00300) and the University of Toronto's Medicine by Design initiative, which receives funding from the Canada First Research Excellence Fund (CFREF). The research was also supported by the National Natural Science Foundation of China (Grant Nos. 11774437 and 61975243) and Local Innovative and Research Teams Project of Guangdong Pearl River Talents Program (Grant No. 2017BT01X121). The authors acknowledge the support from the Centre for Nanostructure Imaging at the Department of Chemistry, University of Toronto for assistance in collecting SEM images, and the Centre for Research and Applications in Fluidic Technologies (CRAFT) for assistance in micromotor fabrication. Finally, we thank Ms. Unė G. Būtaitė (Univ. Glasgow), Dr. Jonathan M. Taylor (Univ. Glasgow), Dr. David B. Phillips (Univ. Exeter), and Dr. Ian Swyer (Univ. Toronto) for fruitful discussions. S. Zhang acknowledges the RBC Post-Doctoral Fellowship program. A.R.W. acknowledges the Canada Research Chair (CRC) program.

\section{Author contributions}

S.Z. conceived the idea. Y.C. and Y.Z. deposited the a-Si:H materials. S.Z. and R.P. built the simulation model and ran the simulations. S.Z. and M.D.C. cultured the cells and prepared the samples. S.Z., M.E., and W.L. fabricated the micromotors and the OET devices. S.Z., M.E., J.P., W.L., and A.N. carried out the experiments. S.Z. and A.R.W. wrote the manuscript. All authors discussed the results and commented on the manuscript. S.Y., X.L., S.L.N., and A.R.W. coordinated and supervised the project.

\section{Competing interests}

The authors declare no competing interests.

\section{Additional information}

Supplementary information The online version contains supplementary material available at https://doi.org/10.1038/s41467-021-25582-8.

Correspondence and requests for materials should be addressed to Aaron R. Wheeler

Peer review information Nature Communications thanks the anonymous reviewers for their contribution to the peer review of this work.

Reprints and permission information is available at http://www.nature.com/reprints

Publisher's note Springer Nature remains neutral with regard to jurisdictional claims in published maps and institutional affiliations.

Open Access This article is licensed under a Creative Commons Attribution 4.0 International License, which permits use, sharing, adaptation, distribution and reproduction in any medium or format, as long as you give appropriate credit to the original author(s) and the source, provide a link to the Creative Commons license, and indicate if changes were made. The images or other third party material in this article are included in the article's Creative Commons license, unless indicated otherwise in a credit line to the material. If material is not included in the article's Creative Commons license and your intended use is not permitted by statutory regulation or exceeds the permitted use, you will need to obtain permission directly from the copyright holder. To view a copy of this license, visit http://creativecommons.org/ licenses/by/4.0/

(C) The Author(s) 2021 